\title{
Visual Attention to the Periphery Is Enhanced in Congenitally Deaf Individuals
}

\author{
D. Bavelier, ${ }^{1}$ A. Tomann, ${ }^{1}$ C. Hutton, ${ }^{2}$ T. Mitchell, ${ }^{3}$ D. Corina, ${ }^{4}$ G. Liu, ${ }^{5}$ and H. Neville ${ }^{3}$ \\ 1 University of Rochester, Brain and Cognitive Sciences, Rochester, New York 14627-0268, ${ }^{2}$ The Wellcome Department of \\ Cognitive Neurology, Institute of Neurology, London WC1N 3BG, United Kingdom, ${ }^{3}$ Department of Psychology, University \\ of Oregon, Eugene, Oregon 97403-1227, ${ }^{4}$ University of Washington, Department of Psychology, Seattle, Washington \\ 98125, and 5 Georgetown University, Washington, D.C. 20007
}

\begin{abstract}
We compared normally hearing individuals and congenitally deaf individuals as they monitored moving stimuli either in the periphery or in the center of the visual field. When participants monitored the peripheral visual field, greater recruitment (as measured by functional magnetic resonance imaging) of the motion-selective area MT/MST was observed in deaf than in hearing individuals, whereas the two groups were comparable when attending to the central visual field. This finding indicates an enhancement of visual attention to peripheral visual space in deaf individuals. Structural equation modeling was used to
\end{abstract}

Although there are numerous anecdotal reports of both enhanced and deficient visual skills in congenitally deaf individuals, few studies have systematically characterized visual processing in congenitally deaf individuals free from neurological deficits. A few studies that have investigated changes in the visual system after early auditory deprivation report a specific enhancement of behavioral performance and neural activity in response to visuospatial information, in particular when presented to the peripheral visual field (Neville et al., 1983; Parasnis and Samar, 1985; Neville and Lawson, 1987a,b; Loke and Song, 1991; Reynolds, 1993; Emmorey, 1998; Bosworth and Dobkins, 1999). For example, Neville and collaborators (Neville and Lawson, 1987a,b) reported faster reaction times and larger visually evoked potentials in deaf than in hearing individuals during the processing of brief peripheral stimuli, whereas the behavioral measures and potentials evoked by central stimuli were comparable across populations. Similarly, Loke and Song (1991) reported faster detection times for peripheral, but not central, visual stimuli in deaf than in hearing individuals. Thus, the little available evidence raises the possibility that peripheral processing is modified after early deafness. In the present study, we used the functional magnetic resonance imaging (fMRI) technique to test the hypothesis that allocation of attention to peripheral visual space is specifically enhanced after auditory deprivation, and we used structural equation modeling to characterize how this modulation arises within the visual pathway.

Participants included hearing and deaf individuals who viewed

Received April 28, 2000; revised June 16, 2000; accepted June 16, 2000.

This work was supported by the Charles A. DANA foundation to D.B., by National Institute of Deafness and Other Communicative Disorders Grant 7-RO1DC00481 to H.N., and by Department of Defense Grant DAMD17-93-V-3018 to the Georgetown Institute for Cognitive and Computational Sciences. We are greatly indebted to the students and staff of Gallaudet University for their enthusiastic participation in this project. We thank Dr. C. Buchel, Dr. J. B. Poline, and Dr. A. Pouget for their technical guidance on issues related to path analysis and statistical analyses, and C. Brozinsky for help with manuscript preparation.

Correspondence should be addressed to Dr. Daphne Bavelier, University of Rochester, Brain and Cognitive Sciences, Meliora Hall 270268, Rochester, NY 14627-0268. E-mail: daphne@bcs.rochester.edu.

Copyright $(\subset 2000$ Society for Neuroscience $0270-6474 / 00 / 200001-\bullet \$ 15.00 / 0$ further characterize the nature of this plastic change in the deaf. The effective connectivity between MT/MST and the posterior parietal cortex was stronger in deaf than in hearing individuals during peripheral but not central attention. Thus, enhanced peripheral attention to moving stimuli in the deaf may be mediated by alterations of the connectivity between MT/MST and the parietal cortex, one of the primary centers for spatial representation and attention.

Key words: plasticity; visual attention; motion; deafness; fMRl; structural equation modeling; MT/MST-V5

alternating blocks of static dots and flow fields of moving dots. Motion flow fields are known to efficiently recruit the motion pathway, including the motion-selective area MT/MST. Visual attention was engaged by requiring participants to monitor the display for luminance changes. Changes in visual attention with eccentricity were tested by contrasting runs in which subjects had to monitor luminance changes in the periphery with those in which subjects had to monitor the luminance changes in the center of the visual field. This design allowed us to compare deaf and hearing individuals when visual attention was allocated to different eccentricities.

\section{MATERIALS AND METHODS}

\section{Participants}

Eleven hearing individuals participated, as well as eleven congenitally, genetically deaf individuals who were born to deaf parents and acquired American Sign Language (ASL) as their native language. All participants were right-handed. Deaf subjects had no history of neurological disorder and had a hearing loss of $>90 \mathrm{~dB}$ binaurally. Data from two of the deaf subjects were discarded because of excessive motion during the session for one participant and a misunderstanding of the task for the other. Participants included in the analysis were between the ages of 18 and 27 years (mean age of 23 years in each group); the hearing group included six females and five males, and the deaf group five females and four males.

This article is published in The Journal of Neuroscience, Rapid Communications Section, which publishes brief, peer-reviewed papers online, not in print. Rapid Communications are posted online approximately one month earlier than they would appear if printed. They are listed in the Table of Contents of the next open issue of JNeurosci. Cite this article as: JNeurosci, 2000, 20:RC93 (1-6). The publication date is the date of posting online at www.jneurosci.org.

http://www.jneurosci.org/cgi/content/full/4475 


\section{Experimental Design}

Stimuli. Participants were scanned during seven runs, each lasting $4 \mathrm{~min}$ and $16 \mathrm{sec}$. During all runs, the subjects looked at a fixation point $\left(0.3^{\circ}\right)$ projected on a transparent screen viewed through a mirror fitted onto the head coil. Images were back-projected on the screen by an LCD video projector. Stimuli consisted of an alternation of moving and static blocks using displays composed of $\sim 280$ dots $\left(0.2^{\circ} / \mathrm{dot}\right)$. On the motion blocks, the dots moved radially at a speed of $4.5^{\circ} / \mathrm{sec}$. Half of the dots moved inward and the other half moved outward to avoid motion aftereffects and eye movements. In the first run, subjects passively viewed alternations of motion and static blocks. In these runs, the dots covered the whole field, and no luminance changes occurred. Of the six remaining runs, only three experimental runs are reported in this paper because the other three runs concerned different experimental manipulations. During the runs of interest, participants were asked to monitor the motion and static blocks for abrupt decreases in luminance. These changes consisted of a $65 \%$ decrease in luminance lasting $1 \mathrm{sec}$. This value was selected so that the task could be performed equally well in the central and peripheral locations. Within a run, changes occurred either in the center only $\left(0.4-1.73^{\circ}\right)$, in the near-periphery only $\left(6.66-8^{\circ}\right)$, or over the whole field $\left(0.4-8^{\circ}\right)$ (see Fig. 1).

Behavioral task. At the end of each run, subjects reported the number of blocks that had three or more luminance changes. Thus, this task required the subject to detect luminance changes, to keep track of their frequency within a block (i.e., during the length of a static block or of a motion block), and to keep track of the number of blocks that had three or more changes during the $4 \mathrm{~min}$ and $16 \mathrm{sec}$ duration of a run. Subjects were trained on the stimuli and tasks before the fMRI session. Once in the magnet, subjects were reminded of the task before each run via text that appeared on the screen that was used for stimulus presentation. They were also reminded to maintain fixation on the central cross throughout the run.

\section{Image acquisition and analysis}

MR parameters. The experiment was performed at Georgetown University on a 1.5T Magnetom VISION (Siemens AG, Erlangen, Germany) whole-body MRI system equipped with a head volume coil. Multislices T2*-weighted fMRI images were obtained with echo-planar imaging (EPI) using a tilted coronal orientation, chosen to align the slices parallel to the participant's calcarine fissure [echo time (TE) $=40 \mathrm{msec}, 64 \times 64$; field of view $(\mathrm{FOV})=224,20$ slices, $5 \mathrm{~mm}$ thickness with $10 \%$ gap, i.e., $3.5 \times 3.5 \times 5.5 \mathrm{~mm}^{3}$ voxel size; repetition time $(\mathrm{TR})=4 \mathrm{sec}, 64$ time points: four time points of a blank screen to eliminate magnetic saturation effects followed by six cycles; each cycle consisted of five time points of the static display and five time points of the motion display]. T2weighted Turbo-Spin Echo matched to the EPI images (TE $=99.0$, $0.85 \times 0.875 \times 5 \mathrm{~mm}$ with $10 \%$ gap) and three-dimensional T1-weighted SPGR volumes were used to define the anatomical and functional localization of the motion area MT/MST in the 11 hearing and 9 deaf subjects. Additionally, the SPGR volumes were required to spatially normalize the functional images as a preprocessing step for the structural equation modeling (see Structural equation modeling analysis below).

Region of interest analysis. Data from each run were realigned using SPM96. Data with motion artifacts $>1.5^{\circ}$ in rotation or half the voxel size in translation were discarded (one deaf subject); the remaining data were corrected for motion (SPM 96). No spatial smoothing was applied to the data because only voxel level inferences were of interest. Low-frequency confounds were removed using a high-pass filter, and the data were temporally smoothed with a $2.8 \mathrm{sec}$ Gaussian kernel. The data were then analyzed within each subject by computing the temporal correlation between the MR signal and a reference function (two temporal basis functions in SPM96) for each voxel. As is standard in analyses using SPM, voxels that did not survive an initial probability threshold of $p<$ 0.001 for the omnibus test ( $F$ ratio) were discarded from further analyses. Voxels were considered active if they reached $p<0.01$ (uncorrected) for the contrast of interest using the fixed effect statistics of SPM96.

After previous fMRI studies, area MT/MST was defined on the basis of both anatomical and functional criteria. Activation from the first run, in which subjects passively observed alternations of moving and static dots, was used for identifying the location of MT/MST by selecting the area of most activation in the inferior part of the lateral occipital lobe. As has been reported in previous studies, MT/MST was localized in most subjects at the temporo-occipital junction and in particular near the intersection of the anterior occipital sulcus and the lateral occipital sulcus (Zeki et al., 1991; Watson et al., 1993). Although the exact location of MT/MST within these sulci varied across subjects, all subjects displayed the most robust motion-related activation in these sulci. The voxel with the highest activation at that lateral position was determined to be the center of MT/MST activation. The size of MT/MST activation was then determined by including all active voxels connected to that point of highest activity (as long as they fell within the boundaries of the anatomical definition of MT/MST). It is worth noting that the analysis parameters used were such that MT/MST activation was spatially distinct from that of other visual areas in all but one subject (deaf). Although this may have resulted in a smaller MT/MST volume than that reported in other studies, it ensured that MT/MST activation was well circumscribed and minimally contaminated by other nearby visual areas. This point is important because it is unknown whether other visual areas also reorganize after early auditory deprivation.

Activation was delineated for each participant, using an interactive region definition program implemented in Matlab (Mathworks, Natick, MA). The program outputs the number of voxels, percentage signal change, and phase of the significantly active voxels (as determined by the fixed effect statistics of SPM96; see above) included in the user-defined regions. Standard ANOVAs were performed on the extent of the activation and on percentage signal change. Separate analyses of these two variables were conducted because it is unclear which of these measures best indexes functional changes in fMRI, especially when comparing group populations and/or assessing plastic changes. Percentage change analyses revealed no effect of population; these analyses will not be discussed further (the percentage changes observed across conditions and populations were all of the order of $2 \%$ ). Although the first run (passive looking) was designed to be used as a localizer and not as an experimental condition, it is worth noting that there was no significant population or hemisphere effects in these runs. The size of MT/MST appeared larger for deaf than for hearing individuals $\left(572 \mathrm{~mm}^{3}\right.$ vs 419 $\left.\mathrm{mm}^{3}\right)$; however, this difference was not robust $(p>0.4)$ because of a great variability in the deaf group under these conditions.

\section{Structural equation modeling analysis}

High-resolution anatomical SPGR volumes were used to spatially normalize the functional images as a preprocessing step for the structural equation modeling. Because these anatomical images were collected for only seven deaf subjects, the structural equation modeling analysis was also restricted to seven hearing subjects so that the maximum power of the analysis could be maintained with equal sample sizes. The same seven hearing participants were used for all structural equation modeling analyses and were selected to have the highest path coefficient between MT/MST and posterior parietal (PP) region in the peripheral condition. Note that because our hypothesis was to test for greater path coefficients in deaf than in hearing in the peripheral condition, this selection criterion biased the analysis against our hypothesis.

The structural equation modeling was composed of unidirectional connections from early visual cortex (V1/V2) to motion areas MT/MST, and from $\mathrm{MT} / \mathrm{MST}$ to the $\mathrm{PP}$ region within each hemisphere. Image processing and statistical analyses were performed using SPM96 (Friston et al., 1995, 1996; Worsley and Friston, 1995) followed by structural equation modeling as described in Buchel and Friston (1997). The realigned, spatially normalized data were smoothed using an $8 \mathrm{~mm}$ full-width at half-maximum isotropic Gaussian kernel and tested for activation effects. Each subject was analyzed individually. Each subject's structural MRI was used to identify V1/V2, MT/MST, and the posterior parietal cortex. These brain areas were localized using previous knowledge of functional activity and anatomy as described in Buchel and Friston (1997). The map of $Z$ statistics comparing moving with static stimuli was then used to define the activation within each brain area by centering a region of interest (ROI) on the most significant voxel $(p<$ 0.05 uncorrected). The same analysis as in Buchel and Friston (1997) was used except for the size of the ROI, which was increased to accommodate the use of slightly larger voxels in our study $(10 \mathrm{~mm}$ instead of $8 \mathrm{~mm})$. In a few cases, activation in the posterior parietal cortex did not survive a corrected threshold of $p<0.05$. The parietal ROI was then defined using the subject's anatomy. This occurred in the following number of hemispheres for each population: deaf: attend-center $=$ four left and two right, attend-periphery $=0$ left and one right, full-field $=0$ left and three right; hearing: attend-center = two left and one right, attend-periphery = two left and five right, and full-field = two left and five right.

In each subject, a representative time-series was created for each region by taking the first eigenvector of all the voxel time-series within the region of interest. The eigenvectors corresponding to the same region were normalized to zero mean and unit variance and then combined across subjects in each condition (attend-center, attend-periphery, fullfield). This resulted in a time-series based on 420 observations (six cycles of 10 time points for seven subjects) for each region and each condition.

Path coefficients were estimated by fitting the structural equation model to the interregional covariances within each group. A $\chi^{2}$ value was obtained that represented the goodness-of-fit of the model to the data. A stacked model analysis was used to assess the significance of the differences in path coefficients between deaf and hearing. This approach statistically compares the $\chi^{2}$ goodness-of-fit values obtained from two different models (McIntosh and Gonzalez-Lima, 1994; Buchel and Friston, 1997). A null or restricted model, in which the paths from V1/V2 to $\mathrm{MT} / \mathrm{MST}$ or from MT/MST to PP were constrained to be equal between the groups, was compared with a free model in which all the path coefficients could differ. The significance of the $\chi^{2}$ difference was assessed using the $\chi^{2}$ distribution with $n$ degrees of freedom, where $n$ is equal to the difference in the numbers of degrees of freedom in each model (Bollen, 1989). In our case, one path was constrained to be equal 
Table 1. Each run comprised alternating blocks of static dots and moving dots

\begin{tabular}{llc} 
& Hearing & Deaf \\
\hline Attend-periphery & $91(9)$ & $100(0)$ \\
Attend-center & $91(9)$ & $78(15)$ \\
Full-field & $91(9)$ & $89(11)$
\end{tabular}

Subjects were asked to pay attention to brief luminance changes and to report the number of blocks with three or more changes. The percentage of runs in which subjects accurately reported the number of blocks with three or more changes is given for each population as a function of the attentional condition.

in the null model compared with no constraints in the free model so that $n=1$. A significant $\chi^{2}$ difference $(p<0.05)$ indicates that the free model in which path coefficients are allowed to differ gives an improved fit. The path coefficients corresponding to a significant $\chi^{2}$ difference can therefore be considered significantly different (Buchel and Friston, 1997; Buchel et al., 1999).

It is important to note that changes in connectivity are not directly related to changes in extent of activation. Although the extent of activation indexes the robustness of the activation within each brain area, structural equation modeling measures the covariance between the fMRI time courses of different brain areas. The present study illustrates this fact because analyses of the extent activation in areas V1/V2 and PP revealed no differences between deaf and hearing $(p>0.06)$, unlike the results reported below for MT/MST.

\section{RESULTS}

\section{Behavioral results}

The task was designed to be equally easy at all spatial locations so that participants could successfully comply with the task demands. Accordingly, participants' performance was high and equivalent for the two groups (89\% correct for deaf and 91\% correct for hearing; an ANOVA with population and attentionlocation as factors performed on the percentage correct response revealed no significant group differences: all $p$ values $>0.4$ ) (Table $1)$. Thus any observed differences in brain activity may not be easily attributed to group differences in task difficulty.

\section{MT/MST recruitment as a function of the location of attention}

The literature is now rich with examples of increases in MT/MST activation when more visual attention is allocated to the processing of moving stimuli (Beauchamp et al., 1997; O'Craven et al., 1997; Rees et al., 1997; Treue and Martinez Trujillo, 1999). Thus, if deaf individuals have enhanced attentional resources in the periphery, they should display a greater recruitment of MT/MST in the peripheral condition. Analyses of the extent of activation in MT/MST (see Fig. 2A) as a function of population, hemisphere, and attention-location (central/peripheral) revealed a significant population by attention-location interaction $\left(F_{(1,18)}=10.6, p<\right.$ $0.004)$, which reflected sensitivities to the location of attention opposite in the two populations (see Fig. $2 B$ ). Separate analyses of the central and peripheral conditions revealed no group difference for the attend-center condition $(p>0.3)$ but a main effect of population in the attend-periphery condition $\left(F_{(1,18)}=\right.$ $6.0, p<.024)$. Thus, although MT/MST recruitment was comparable across populations when the center of the visual field was monitored, deaf individuals displayed greater MT/MST activation than hearing subjects when the peripheral visual field was monitored. More importantly, the presence of an interaction between population and center/periphery indicates larger population differences under peripheral than central attention, demonstrating a specific functional reorganization after auditory deprivation.

\section{MT/MST and eye movements}

Recent investigations suggest that eye movements lead to the recruitment of MT/MST (Petit and Haxby, 1999); thus another

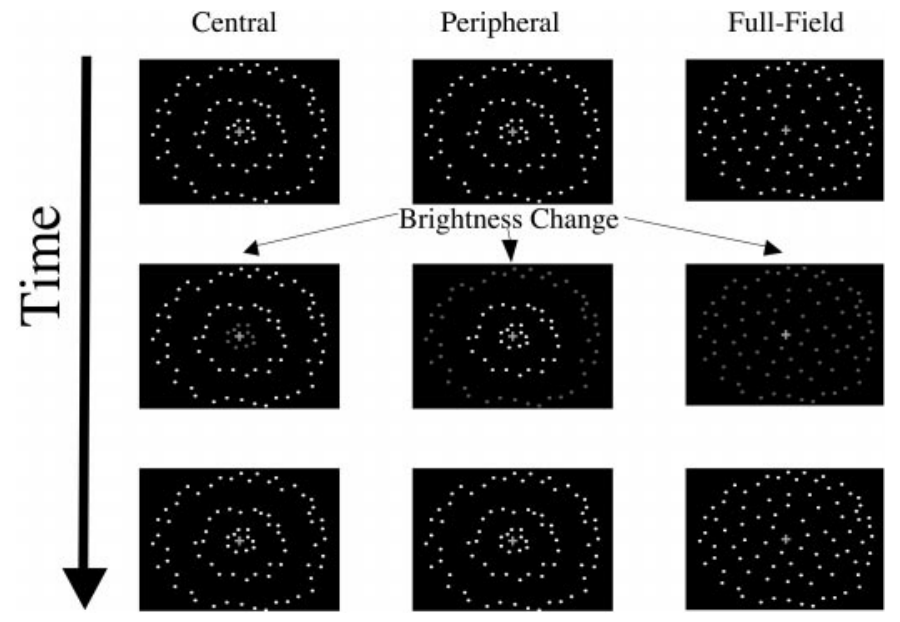

Figure 1. Schematic representation of the stimuli used for the central, peripheral, and full-field conditions. Participants were asked to monitor luminance changes either in the center or in the near-periphery ring. In the attend-center conditions, luminance changes occurred only in the center ring; in the attend-periphery condition, luminance changes occurred only in the near-periphery ring.

interpretation of this finding is that deaf individuals were more likely than hearing to move their eyes in the peripheral condition. Subjects were reminded to fixate throughout the presentation, and the stimuli moved both inward and outward to avoid tracking; however, eye movements were not recorded during the fMRI runs. To check for possible artifacts, three subjects from each population viewed the same stimuli as in the magnet while eye movements were recorded with an ASL 504-remote camera system. Although these data indicated some eye movements, there was no evidence for more eye movements during the motion than the during static block, suggesting that it is unlikely that MT/MST activation is caused by eye movements. To verify that the population differences observed in MT/MST are unlikely to result from differences in eye movement patterns, activation within the brain areas associated with eye movements (i.e., frontal eye field) was assessed in each population using the same analysis technique as for MT/MST. If eye movements are responsible for the population difference observed in MT/MST, similar population differences should be observed in the frontal eye field. Recent investigations of saccadic eye movements and pursuit eye movements indicate that they result in reliable recruitment of the precentral gyrus extending from the central sulcus to the precentral sulcus (Corbetta, 1998). In each subject, the central sulcus, superior frontal sulcus, and inferior precentral sulcus were delineated, and any activation falling in gray matter within the region bounded by these sulci was recorded. ANOVAs with population and either central/peripheral condition or central/peripheral/full-field condition as factors revealed no significant effect of population (all $p$ values including population as a factor $>0.14$ ). The absence of any population effect in the frontal eye field indicates that MT/MST differences cannot be easily interpreted in terms of artifacts from eye movements.

\section{Full-field condition}

Our design also included a third condition (full-field) in which participants viewed a display of dots randomly distributed over the whole visual field, rather than restricted to three rings (Fig. 1), and had to monitor luminance changes that occurred over the whole field at once. MT/MST activation was comparable across populations in this condition $(p>0.95)$. This finding suggests that the difference between deaf and hearing individuals ob- 
Figure 2. A, Example of MT/MST activation in three deaf and three hearing participants shown in the orientation in which the data were collected (i.e., cuts parallel to the calcarine). $B$, Extent of the activation in MT/MST (in $\mathrm{mm}^{3}$, with SEM) as a function of the experimental conditions in each population (for details, see Materials and Methods, ROI analysis). Deaf individuals displayed greater recruitment of MT/MST than hearing controls when monitoring peripheral stimuli. $C$, Extent of the activation in MT/ MST (in $\mathrm{mm}^{3}$, with SEM) as a function of hemispheres in each population. Lateralization of the MT/MST activation differed in deaf and hearing individuals because of a larger recruitment of left MT/ MST in the deaf.
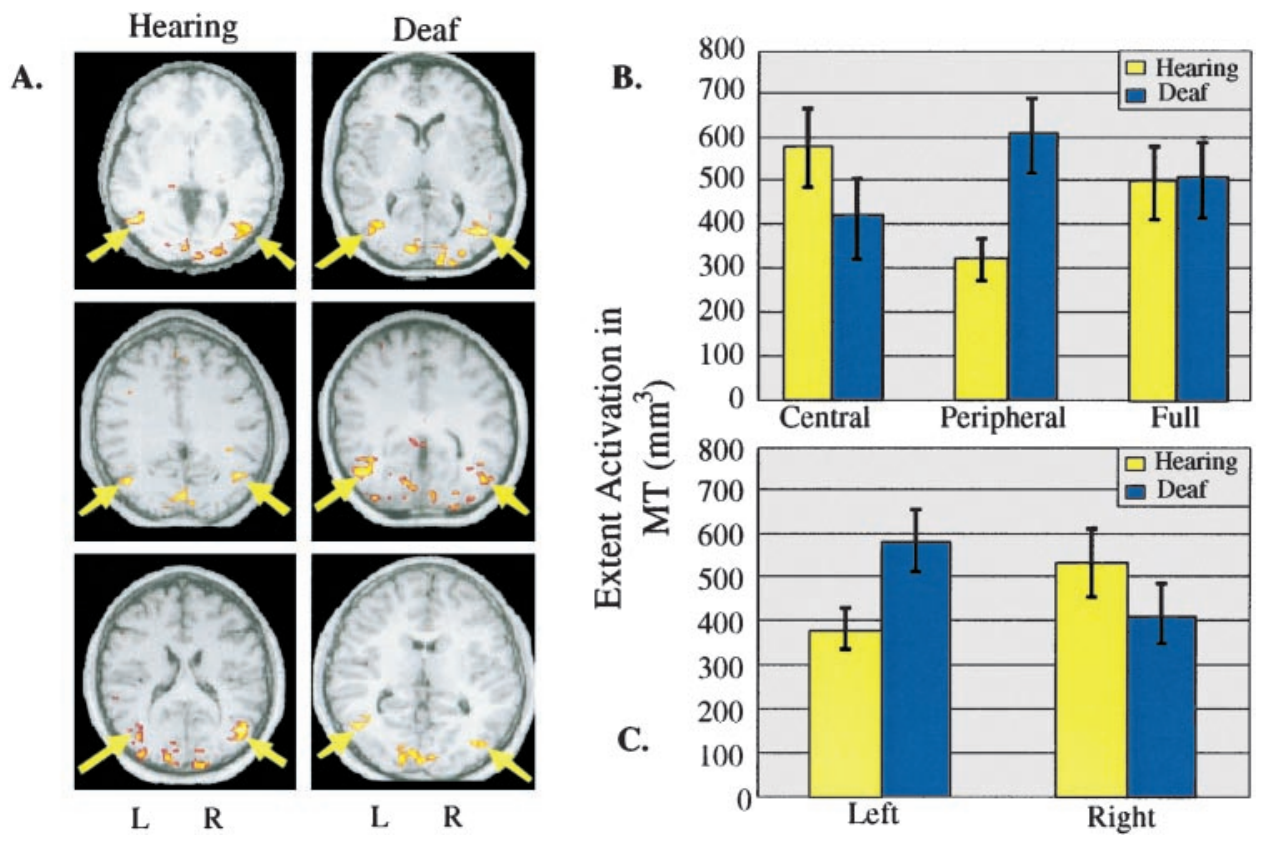

served during the peripheral runs is unlikely to be mediated by the enhancement of an automatic response whenever luminance levels are varied in the periphery. Rather, the lack of a population difference in both the attend-central and full-field conditions is compatible with the view that changes in endogenous attention to peripheral stimuli mediate the population difference observed in the peripheral condition. Indeed, in the full-field condition, participants knew ahead of time that changes would occur simultaneously over the central and peripheral field and so could perform the task by endogenously directing their attention only to the central portion of the visual field. Although it will be important in further studies to assess the relative contribution of exogenous and endogenous attention in the population differences described above, results from the full-field condition highlight the importance of endogenous attention.

\section{Discussion of the peripheral/central difference}

The finding of greater population differences in MT/MST recruitment during the peripheral attention condition than during the central and full-field conditions indicates a specific modulation of attention to peripheral moving stimuli in the deaf. This finding provides one plausible functional basis for the behavioral and event-related potential effects described previously by Neville and Lawson (1987a,b). In their study, both behavioral and event-related potential data showed that deaf subjects performed faster and exhibited higher sensitivities when monitoring the direction of motion of a moving square in the periphery but were comparable with hearing controls for central motion. The enhanced peripheral processing reported by these authors appeared to be caused by early deafness rather than early exposure to sign language, because it was observed in deaf signers but not in hearing native signers (Neville and Lawson, 1987c). Thus, although the present experiment did not include a group of hearing signers, these earlier findings indicate that the peripheral enhancement reported above is likely to be caused by early auditory deprivation rather than the use of sign language.

\section{Lateralization of MT/MST activation}

Another result of interest concerns a marked group difference in hemispheric lateralization. The MT/MST activation was larger in the left MT/MST of deaf individuals but tended to be larger in the right MT/MST of hearing individuals (Fig. 2C). Indeed, an ANOVA of the extent of MT/MST activation (including central, peripheral, and full-field) indicated a population by hemisphere interaction $\left(F_{(1,18)}=7.9, p<0.012\right)$ because of a left hemisphere bias for deaf subjects and the opposite trend for hearing individuals. This shift in lateralization was observed in all attentional conditions (no population by hemisphere by condition interaction: $p>0.08$ ). This change in MT/MST lateralization mirrors population differences in lateralization that have been documented in other studies. These studies have reported a right visual field/left hemisphere dominance for motion processing in the deaf, whereas hearing controls display a bilateral pattern with possibly a slight left visual field bias for motion processing (Neville and Lawson, 1987a,b; Bosworth and Dobkin, 1999). Interestingly, in contrast to the enhancement of peripheral processing, these authors observed the same left hemisphere advantage in hearing native signers, suggesting that this laterality difference is more likely to be caused by early exposure to sign language than to auditory deprivation per se.

\section{Functional connectivity in the motion pathway as a function of the location of attention}

To further characterize the altered organization observed in the deaf, we used structural equation modeling, which estimates the strength of cortical connections between areas. Using structural equation modeling, we compared the effective connectivity within the network of areas recruited during attention to moving stimuli in deaf and hearing participants. We based our analysis on the known anatomical connectivity within primate posterior visual regions during motion processing (Fellman and Van Essen, 1991; Distler et al., 1993). As in previous human brain imaging research, we tested the occipito-temporo-parietal network, including three regions of interest: early visual areas (V1/V2), the motion-sensitive area MT/MST, and part of the PP cortex (McIntosh and Gonzalez-Lima, 1994; Beauchamp and DeYoe, 1996; Buchel and Friston, 1997). The anatomical model based on this network was fit to the covariance structure of the time series extracted from the regions of interest as in Buchel and Friston (1997) (see Materials and Methods). The model parameters are path coefficients that provide an estimate of the effective connec- 


\begin{tabular}{|c|c|c|c|c|c|c|}
\hline & \multicolumn{2}{|c|}{ Attend-periphery $\mathrm{V} 1 \rightarrow \mathrm{MT} \rightarrow \mathrm{PP}$} & \multicolumn{2}{|c|}{ Attend-center $\mathrm{V} 1 \rightarrow \mathrm{MT} \rightarrow \mathrm{PP}$} & \multicolumn{2}{|c|}{ Full-field V1 $\rightarrow \mathrm{MT} \rightarrow \mathrm{PP}$} \\
\hline \multicolumn{7}{|l|}{ Left } \\
\hline Hearing & 0.77 & 0.56 & 0.77 & 0.56 & 0.80 & 0.58 \\
\hline Deaf & 0.82 & 0.68 & 0.82 & 0.52 & 0.82 & 0.64 \\
\hline$\chi^{2}$ & 1.1 & 4.9 & 1.0 & 0.4 & 0.2 & 1.27 \\
\hline ( $p$ value) & $(p>0.3)$ & $(p<0.03)$ & $(p>0.3)$ & $(p>0.5)$ & $(p>0.6)$ & $(p>0.25)$ \\
\hline \multicolumn{7}{|l|}{ Right } \\
\hline Hearing & 0.77 & 0.38 & 0.78 & 0.65 & 0.80 & 0.55 \\
\hline Deaf & 0.81 & 0.62 & 0.78 & 0.62 & 0.83 & 0.52 \\
\hline$\chi^{2}$ & 0.8 & 16.6 & 0 & 0.3 & 0.3 & 0.3 \\
\hline ( $p$ value) & $(p>0.3)$ & $(p<0.001)$ & $(p>0.9)$ & $(p>0.5)$ & $(p>0.5)$ & $(p>0.5)$ \\
\hline
\end{tabular}

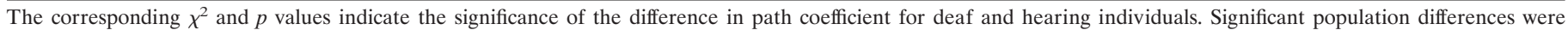
observed only in the peripheral attention condition for the MT/MST to PP connection.

tivity between the regions. The path coefficients were comparable across groups for all conditions except the peripheral attention condition (Table 2). During the attend-periphery condition, deaf and hearing participants had equivalent effective connectivity between V1 and MT/MST, but the effective connectivity between $\mathrm{MT} / \mathrm{MST}$ and PP was increased in the deaf as compared with the hearing in both hemispheres. In line with previous reports that enhanced processing is mediated by increased connectivity between the network of areas defined by the task (Buchel et al., 1999; McIntosh et al., 1999), this finding suggests an enhancement of peripheral attention to motion in deaf individuals. More importantly, because alterations of the motion pathway were restricted to the MT/MST-PP connection, the structural equation modeling analysis indicates that this enhancement is specific to the higher stages of visual processing.

\section{DISCUSSION}

Taken together, these results demonstrate marked and specific changes in the organization of the motion pathway in congenitally deaf individuals. In all viewing conditions, deaf individuals, unlike hearing subjects, displayed left lateralized MT/MST activation. This left lateralization has been hypothesized to result from the temporal coincidence of motion information and linguistic comprehension in signed languages (Neville and Lawson, 1987a,b; Bosworth and Dobkins, 1999). When attending to the periphery, deaf individuals displayed a larger recruitment of the motion pathway than hearing individuals, whereas no population differences were noted when attending to the center. This result implies that the representation of peripheral space is more dependent on, and modifiable by, early auditory deprivation than is the representation of central visual space. A recent study of congenitally blind individuals reports a parallel pattern for central and peripheral auditory attention (Roder et al., 1999). Further research is required to investigate the possible mechanisms that might mediate this specific type of change across both visual and auditory modalities. In particular, the studies available today cannot distinguish between (1) an increased number of neurons that code weakly for the peripheral field in the deaf or the blind and are efficiently recruited under the modulatory influence of peripheral attention and (2) an equal number of peripheral neurons in experimental and control populations but differences in gain modulation under central and peripheral attention in the different populations. Although the present study cannot separate these alternatives, it establishes that the mechanism(s) at play includes alterations of the connectivity between earlier sensory areas and the parietal cortex, one of the primary centers for spatial representation and attention.

\section{REFERENCES}

Beauchamp M, DeYoe E (1996) Brain areas for processing motion and their modulation by selective attention. NeuroImage 3[Suppl]:S245.

Beauchamp M, Cox R, DeYoe E (1997) Graded effects of spatial and featural attention on human area MT and associated motion processing areas. J Neurophysiol 78:516-520.

Bollen K (1989) Structural equations with latent variables. New York: Wiley.

Bosworth R, Dobkins K (1999) Left hemisphere dominance for motion processing in deaf signers. Psychol Sci 10:256.

Buchel C, Friston KJ (1997) Modulation of connectivity in visual pathways by attention: cortical interactions evaluated with structural equation modelling and fMRI. Cereb Cortex 7:768-778.

Buchel C, Coull JT, Friston KJ (1999) The predictive value of changes in effective connectivity for human learning. Science 283:1538-1541.

Corbetta M (1998) Frontoparietal cortical networks for directing attention and the eye to visual locations: identical, independent, or overlapping neural systems? Proc Natl Acad Sci USA 95:831-838.

Distler C, Boussaoud D, Desimone R, Ungerleider LG (1993) Cortical connections of inferior temporal area TEO in macaque monkeys. J Comp Neurol 334:125-150.

Emmorey K (1998) The impact of sign language use on visuospatial cognition. In: Psychological perspectives on deafness (Marschark M, Clark MD, eds), pp 19-52. Mahwah, NJ: Lawrence Erlbaum Associates.

Fellman DJ, Van Essen DC (1991) Distributed hierarchical processing in the primate cerebral cortex. Cereb Cortex 1:1-47.

Friston KJ, Holmes AP, Poline J-B, Grasby PJ, Williams SCR, Frackowiak RSJ, Turner R (1995) Analysis of fMRI time-series revisited. NeuroImage 2:45-53.

Friston KJ, Holmes AP, Ashburner J, Poline J-B (1996) SPM96. Available at http://www.fil.ion.ucl.ac.uk/spm.

Loke WH, Song S (1991) Central and peripheral visual processing in hearing and nonhearing individuals. Bull Psychonom Soc 29:437-440.

McIntosh AR, Gonzalez-Lima F (1994) Structural equation modelling and its application to network analysis in functional brain imaging. Hum Brain Mapp 2:2-22.

McIntosh AR, Rajah MN, Lobaugh NJ (1999) Interactions of prefrontal cortex in relation to awareness in sensory learning. Science 284:1531-1533.

Neville HJ, Lawson DS (1987a) Attention to central and peripheral visual space in a movement detection task: an event-related potential and behavioral study. I. Normal hearing adults. Brain Res 405:253-267.

Neville HJ, Lawson DS (1987b) Attention to central and peripheral visual space in a movement detection task: an event related potential and behavioral study. II. Congenitally deaf adults. Brain Res 405:268-283.

Neville HJ, Lawson DS (1987c) Attention to central and peripheral visual space in a movement decision task. III. Separate effects of auditory deprivation and acquisition of a visual language. Brain Res 405:284-294.

Neville HJ, Schmidt AL, Kutas M (1983) Altered visual-evoked potentials in congenitally deaf adults. Brain Res 266:127-132.

O'Craven KM, Rosen BR, Kwong KK, Treisman A, Savoy RL (1997) 
Voluntary attention modulates fMRI activity in human MT-MST. Neuron 18:591-598.

Parasnis I, Samar VJ (1985) Parafoveal attention in congenitally deaf and hearing young adults. Brain Cognit 4:313-327.

Petit L, Haxby JV (1999) Functional anatomy of pursuit eye movements in humans as revealed by fMRI. J Neurophysiol 81:463-471.

Rees G, Frith CD, Lavie N (1997) Modulating irrelevant motion perception by varying attentional load in an unrelated task. Science 278:1616-1619.

Reynolds HN (1993) Effects of foveal stimulation on peripheral visual processing and laterality in deaf and hearing subjects. Am J Psychol 106:523-540

Roder B, Teder-Salejarvi W, Sterr A, Rosler F, Hillyard S, Neville H
(1999) Improved auditory spatial tuning in blind humans. Nature 400:162-166.

Treue S, Martinez Trujillo J (1999) Feature-based attention influences motion processing gain in macaque visual cortex. Nature 399:575-579.

Watson JDG, Myers R, Frackowiak RS, Hajnal JV, Woods RP, Mazziotta JC, Shipp S, Zeki S (1993) Area V5 of the human brain: evidence from a combined study using positron emission tomography and magnetic resonance imaging. Cereb Cortex 3:79-94.

Worsley KJ, Friston KJ (1995) Analysis of fMRI time series revisitedagain. NeuroImage 2:173-181.

Zeki S, Watson JD, Lueck CJ, Friston KJ, Kennard C, Frackowiak RS. (1991) A direct demonstration of functional specialization in human visual cortex. J Neurosci 11:641-649. 